This is a self-archiving document (manuscript version):

Subjectively estimated vs. objectively measured adaptation to driving simulators - Effects of age, driving experience, and previous simulator adaptation

By: Brandtner, Annika; Liebherr, Magnus; Schweig, Stephan; Maas, Niko; Schramm, Dieter; Brand, Matthias

Published in: Transportation Research Part F: Traffic Psychology and Behaviour

Volume 64, July 2019, Pages 440-446 (Please be aware: Page numbering in this manuscript can be different to published version!)

Publisher: Elsevier | Year: 2019

DOI: 10.1016/j.trf.2019.05.019

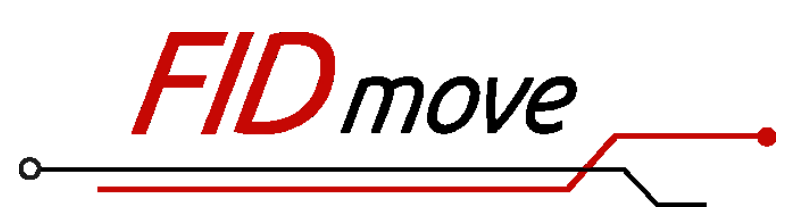

Brought to you by the Scientific Information Service for Mobility and Transport Research

Fachinformationsdienst Mobilitäts- und Verkehrsforschung

Website: www.fid-move.de

Repository: publish.fid-move.de

Contact: publish@fid-move.de

(C) 2021. This manuscript version is made available under the CC-BYNC-ND 4.0 license. 


\title{
Subjectively estimated vs. objectively measured adaptation to driving simulators - Effects of age, driving experience, and previous simulator adaptation
}

\author{
Annika Brandtner ${ }^{\mathrm{a}, *}$, Magnus Liebherr ${ }^{\mathrm{a}}$, Stephan Schweig ${ }^{\mathrm{b}}$, Niko Maas ${ }^{\mathrm{b}}$, Dieter Schramm ${ }^{\mathrm{b}}$, \\ Matthias Brand ${ }^{a}$ \\ a University of Duisburg-Essen, Department of General Psychology: Cognition, Duisburg, Germany \\ ${ }^{\mathrm{b}}$ University of Duisburg-Essen, Department of Mechatronics, University of Duisburg-Essen, Germany \\ * Corresponding author.
}

\section{A B S T R A C T}

Objective: The present study aims to investigate whether drivers' age and their experience with driving simulators could explain differences between a subjective estimation of system adaptation and a respective objective systematic measurement.

Background: Assessing valid measurements in driving simulators causes concern because driving simulators are not yet as realistic as real on-road driving scenarios. Common methods like pre-defined training sessions and self-appraisals of simulator adaptation might therefore be insufficient to ensure actual valid data. Hence, influential variables on this discrepancy are investigated.

Method: In total, $N=203$ drivers participated in a training session and a subsequent testing session in a close-to-production driving simulator. Subjective adaptation was estimated by the drivers and an objective adaptation value was gathered on the basis of driving accuracy. The discrepancy between these two measures was calculated and related to age, self-reported driving experience and occurrence of previous adaptation.

Results: Subjective adaptation was significantly faster than objective adaptation but neither drivers' age, experience, nor previous adaptation could explain this discrepancy. Discussion: Results indicate that younger and older drivers likewise underestimate the time needed for adaptation. Measuring a subjective point of adaptation seems to be an insufficient measure to ensure simulator validity when assessing both older and younger drivers.

\author{
Keywords: \\ Driving simulator \\ Simulator adaptation \\ Aging \\ Self-appraisal
}

\section{Introduction}

Simulators are frequently used for investigating technical innovations, individual differences in accepting technical environments, as well as the influence of external factors on driving performance. While real-world studies are most often characterized by an increased complexity and the appearance of various difficulties, simulators offer the possibility to examine relevant issues on a highly standardized, but also cost and time efficient level (Desmond \& Matthews, 1997). Furthermore, the risk of accidents or injuries that might occur in real-world studies, can be excluded by using simulated 
environments. While simulators were originally developed in the context of flying, they are most often used in driving studies nowadays (Weinberg \& Harsham, 2009). In contrast to early simulators that have been very simple and rudimentary, current simulators are greatly developed, aiming to reproduce most possibly realistic behaviors (Bayarri, Fernandez, \& Perez, 1996). Although previous studies showed that drivers may be willing to behave alike in simulators and on real roads (Underwood, Crundall, \& Chapman, 2011), others are still concerned about the validity of the measures collected in driving simulators. For example, Kemeny and Panerai (2003) stated that driving simulators do not provide all of the most relevant visual cues for the driver to feel and behave as in a real driving situation. Moreover, Owsley and McGwin (2010) point out crude visual displaying in driving simulators which do not ensure realistic lightning in simulated driving situations and therefore lead to invalid measurements. Many previous validation studies have therefore tried to find a way of validating simulators which was most commonly implemented by measuring and comparing driving speed behaviors or evaluating if drivers have similar speed performance in a simulator and in on-road situations (Godley, Triggs, \& Fildes, 2002; Klee, Bauer, Radwan, \& Al-Deek, 1999). Results showed that participants tended to drive at slower speed in the simulator, indicating a probable uncertainty towards the virtual reality shown in the simulator. Hence, both studies failed in absolute validity as speed performances differed. A most widely accepted method to ensure realistic driving behavior is the consideration of simulator adaptation (Domeyer, Cassavaugh, \& Backs, 2013), which describes the time by which drivers adjust their existing driving skills to the simulator so that they can effectively control the simulated vehicle and drive it through the simulated environment. This consideration allows people in simulator studies to get comfortable with the system and get used to possible differences between an on-road and simulated environment. Most simulator studies used pre-defined times of 5-15 min (e.g., Broen \& Chiang, 1996; Dorn \& Barker, 2005; Horberry, Anderson, \& Regan, 2006) or pre-defined distances (e.g., Boyle \& Mannering, 2004; Lewis-Evans \& Charlton, 2006) to ensure simulator adaptation. These pre-defined periods are neglecting the individual differences between driving behaviors and are therefore lacking in accuracy. In order to consider the subjective component, other studies have asked their participants about their feelings of comfort (e.g., Maltz \& Shinar, 2004; Salvucci, 2001; Takayama \& Nass, 2008; Ronen \& Yair, 2013). For example, Ronen and Yair (2013) reported a time at which participants felt fully acclimated to the simulator of approximately eleven minutes for curved roads, nine minutes for urban roads, and seven minutes for straight roads. Although the time of pre-defined periods and subjective feelings are reported consistently across several studies, these approaches do not ensure actual adaptation. A more objective method to quantify the time of adaptation resting upon technical data from the driving simulator was established by McGehee, Lee, Rizzo, Dawson, and Bateman (2004). Within their study, the time of adaption was calculated through parameters like steering wheel reversals, magnitude of steering inputs, lane position deviation, frequency components of the lane position and steering inputs which are basically parameters indicating the driving performance in a driving simulator. It is assumed that these parameters are able to detect the approximate time of simulator adaption because compensatory movements made by participants to get along with the simulation are decreasing after a practice period. According to the results of McGehee et al. (2004), both younger and older participants need approximately $240 \mathrm{~s}$ to adapt to the driving simulator after starting to drive within the simulated scenario. In this context, Lee, Cameron, \& Lee (2003) investigated the comparability of the simulated driving performance index and the on-road driving performance index. They found that the simulated driving performance index explains over two thirds of the variability of the on-road performance index and is hence a valid and objective measure of the time of adaptation. Taken together, system adaptation is a highly relevant precondition for valid measures in experimental settings carried out using driving simulators. Next to predefined practice periods that shall ensure adaptation, also subjective estimations and objective measurements of adaptation exist. However, and what is underresearched in previous literature is the comparison of these measures. Further studies working with subjective and objective adaptation to simulators need to be provided with more research and respective findings on the fit of these methods of measurements in order to ensure validity of their data. This study therefore aims at comparing an objective measure of adaptation and the subjective estimation of adaptation within a driving simulator. One former study of Ronen and Yair (2013) found that the sensation of adaptation can give a relatively good indication of adaption for a variety of performance measures. To our best knowledge, this is the only study investigating this comparison and there is yet no study researching the fit of objective and subjective adaptation, and if potential differences can be explained by certain variables. Here, we hold age of the participants, driving experience and their driving expertise in driving simulators to be relevant influential variables. Clarifying this linkage can help to ascertain how much importance needs to be assigned to each of these measures when assessing younger and older participants in a driving simulator. Age is a variable that we assume to have an impact on self-appraisal in virtual realities, because older participants had less time to get used to simulators and are therefore less accurate when self-assessing their driving skills in simulators. Along with the confidence to rate one's driving skills, Matthews \& Moran (1986) found that younger drivers were more confident in their driving ability, which could partly be confirmed by Glendon, Dorn, Davies, Matthews, and Taylor (1996). Therefore, it is assumed that age might also have an effect on the discrepancy deriving from an objective point of adaptation and a subjective estimation of that point. Moreover, it is supposed that participants who already adapted to the same driving simulator in a previous training session might differ from participants who did not experience adaptation in the simulated environment. Drawing on this, Domeyer et al. (2013) found that due to an adaptation to the simulator, motion sickness in the next driving session one day later could be reduced. We therefore suppose that a discrepancy between a subjective estimation of adaptation and an objective measurement of adaptation should be different in drivers that previously adapted to the system to the extent that adapted drivers show less discrepancy between both values. Thus, the following hypotheses are stated: Firstly, we propose that there is a difference between the objective adaptation measured, and the subjective adaptation estimated by the participants. 
This difference is explainable by previous simulator adaptation in that adapted drivers and non-adapted drivers differ in their discrepancy of objective and subjective adaptation. Lastly, we assume that age of the driver and the driving experience as well as previous adaptation to the driving simulator predicts the discrepancy of objective and subjective adaptation.

\section{Methods}

\subsection{Participants}

In total, $N=222$ participants took part in the present study. $N=19$ participants were excluded from calculation as they did not adapt to the driving simulator. They neither adapted objectively, nor did they give a subjective estimation of their adaptation point, supposing that they did not feel comfortable with the driving simulator at any time. Accordingly, there were no values to be compared, wherefore these participants were excluded from further analysis. Hence, $N=203$ participants were included in the study (mean age $=57.37 S D=16.39$ years, ranging from 20 to 89 years, 53 women). Inclusion criteria for the participation were the possession of a driving license and that participants were actively driving a car in their daily lives. Participants who reported neurological or cardio-vascular diseases but also impairments in the ability to see or hear were excluded. Moreover, people aged more than 60 years were tested with the 'DemTect-Test' (Kalbe et al., 2004; Kessler, Calabrese, Kalbe, \& Berger, 2000) and excluded if they showed first signs of dementia (DemTect $<9$ ). The study was performed in accordance with the ethical standards laid down in the Declaration of Helsinki and approved by the local ethics committee at University Duisburg-Essen. All participants provided written informed consent prior to the experiment and were informed that they could end participation at any time without reprisal.

\subsection{Instruments}

To measure the subjective as well as the objective point of adaptation for younger and older participants, we used a static driving simulator. The simulator is a close-to-production vehicle of the compact class which has been extended by force feedback components for the simulation of forces and torques. To create the best possible feeling of a real driving situation, the steering is to be mentioned as one of the most bi-directional interfaces between driver and vehicle. The steering, in particular, is therefore highly important for the feeling of adaptation. The simulator is located in a rectangular container, the simulated environment being projected on the containers' walls. The field of view that was created by the projection is $180^{\circ}$ and therefore includes the entire vehicle front. The side mirrors consist of screens that also represent the environment of the simulated situation. Additionally, a monitor is placed behind the rear window which is used for both the rear view and the reflection in the driving mirror. The representation of the simulated environment includes other road users who interact with the simulated (EGO) vehicle (see Maas, 2017). The inputs of the driver are read by means of the vehicle CAN bus and used as inputs of a complete vehicle simulation (compare Maas, Hesse, Koppers, \& Schramm, 2014) which creates a realistic driving behavior. The given features of the driving simulator create an environment whose usability is physically not different from a series car and therefore creates a realistic driving experience. Still, the visualization is a strong simplification of reality and the missing movements of the driving simulator itself are detrimental to a simulation close to reality. The virtual road scenario is an area of $3 \times 3 \mathrm{~km}$, including several loops that create the impression of an infinite environment. Participants drove on highways that mainly consist of slightly curved, multilane roads. The driving experience of the drivers in this sample was operationalized as the time they possessed their driving license. Hence, the point of time when getting their driver license was used to calculate the number of years that they could possibly gather experience with driving.

\subsection{Data analysis \& statistics}

\subsubsection{Objective and subjective measures of adaptation}

While participants were driving in the simulator, numerous data on their driving performance were collected (e.g. driver's steering behavior, pedal activity etc.). In comparison to earlier experiments (Schweig, Liebherr, Schramm, Brand, \& Maas, 2018), the objective adaptation time was only determined by the compensating steering movements and lane drifts, since pedal activity plays no role on the motorway. Tracking deviation and hectic compensatory movements on the steering wheel are significantly reduced during adaptation. MATLAB was used to calculate and graphically display the square control surface and the time-weighted control surface of the track deviation (Fig. 1). An adaptation can be recognized by a constant average value of the deviations. Smaller fluctuations are caused by lane changes and overtaking operations.

Subjective adaptation was examined by a button that was located next to the driving seat (Fig. 2). Participants were asked to press the button as soon as they felt comfortable with the simulated situation and the reactions of the driving simulator. The time when participants pressed the button was measured.

\subsubsection{Statistical analysis}

Statistical analyses were carried out by using SPSS Statistics 24.0 for Windows (IBM SPSS Statistics, released 2016). Assumptions for the single calculations were checked. A Levene's test revealed no significant deviation from homogeneity of variances $(p=0.718)$ between groups who adapted and did not adapt to the driving simulator in the previous training 


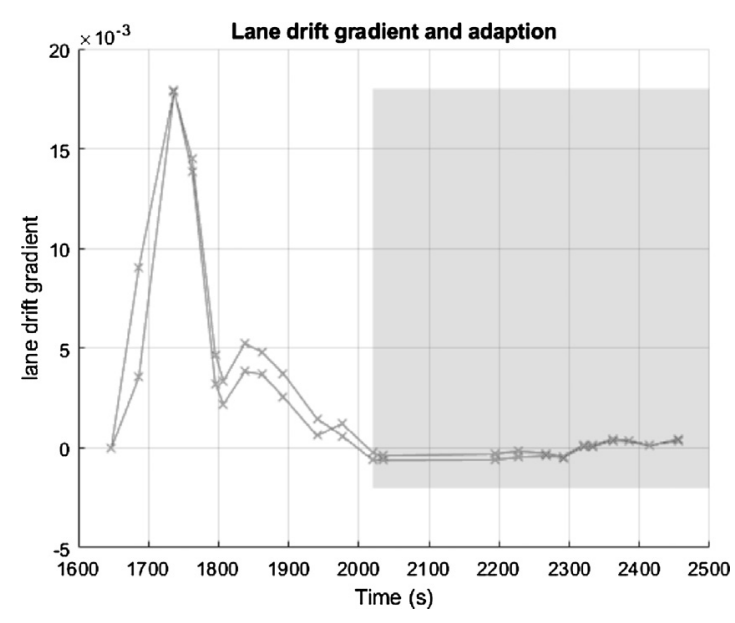

Fig. 1. Lane drift gradients for simulator adaptation of a driver.

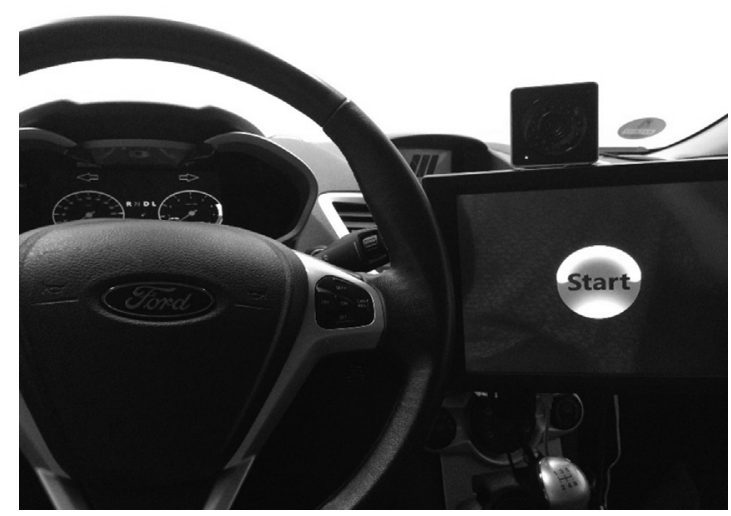

Fig. 2. (Start-) button for subjective point of adaptation.

session. A Durbin Watson statistic addressing the regression analysis revealed a value of 1.4 and is therefore close to a range of 1.5-2.5 which does not give rise for concern. Despite the fact that standardized residuals were not normally distributed $(p<0.001)$ and therefore violated the assumption for a regression analysis, it has still been conducted. Some of the participants had adapted to the system but had not pressed the adaptation-button (see Fig. 2) and vice versa. In order to be still able to provide values in these cases, the point of adaptation was set as the maximum time they spent in the driving simulator. Hence, if a participant spent $1100 \mathrm{~s}$ in the driving simulator, pressed the button after $200 \mathrm{~s}$ but the system did not provide an objective point of adaptation, $1100 \mathrm{~s}$ was set to be the objective adaptation value. This step enabled the calculation of percentage values of each time of adaptation, indicating the point of adaptation expressed as a percentage of the overall time in the simulator. On the basis of this data, a difference between both values could be calculated, resulting in the modulus value of discrepancy between subjective and objective adaptation.

\section{Results}

Of the overall sample of 203 participants, $N=163$ participants pressed the button and therefore gave an estimation of their driving simulator adaptation, $N=141$ participants actually adapted to the system as measured with the algorithm. $N=197$ participants specified the year they attained their driver license wherefore the timespan of driver license possession could be calculated. Descriptive statistics concerning the overall sample are shown in Table 1.

Regarding the first hypothesis, we assumed a difference between objective and subjective adaptation. A paired $t$-test showed a highly significant difference, $t\left(\begin{array}{lll}1 & 0 & 0\end{array}\right)=-3.48, p=0.001$, indicating that the subjective point of adaptation was significantly earlier than the actual, objective point of adaptation. For the question regarding a difference between drivers that adapted and drivers that did not adapt in a previous driving session in the same simulator, an independent $t$-test revealed no significant difference, $t\left(\begin{array}{lll}2 & 0 & 1\end{array}\right)=-0.478, p=0.633$, indicating that a previous training session did not contribute to a better fit of estimated and actual adaptation. In a last step, an exploratory stepwise regression was conducted to identify variables that 


\begin{tabular}{llll}
\hline & Mean & Standard deviation & Maximum \\
\hline Time in the DS (sec) & 847.05 & 116.91 & 618 \\
Subjective adaptation (sec) & 124.59 & 96.72 & 7.25 \\
Objective adaptation (sec) & 211.49 & 216.23 & 4.91 \\
Discrepancy in adaptation (\%) & 49.86 & 37.35 & 0.10 \\
Driver license possession (years) & 38.14 & 16.21 & 963.23 \\
\hline
\end{tabular}

Note. DS = Driving Simulator; Discrepancy in adaptation is measured as the modulus of the subtraction; Driver license possession as measured in years.

possibly contribute to the discrepancy in objective and subjective adaptation. Here, age and the years of driver license possession were entered stepwise into the regression model. The overall model including both predictors does not reach significance $(F(2,196)=2.199, p=0.114)$, indicating that the model has not improved the ability to predict discrepancy in points of adaptation. Table 2 shows the single model parameters.

\section{Discussion}

The aim of the present study was to investigate whether there would be differences in objective and subjective points of adaptation and if such differences would be explainable by a previous adaptation to the same simulator, age of the drivers and the time span of possessing a driver license. Therefore, in the study at hand, an objective, system aggregated point of adaptation was used and compared against a subjective estimation of having adapted to the simulator. Results show that participants rated their point of adaptation as being significantly earlier than their actual objective point of adaptation wherefore the question arises, what could have led to an overestimation, independently of age and driving experience of the drivers or through the difference between drivers who adapted or did not adapt in a previous training session. Towards an explanation of this difference, Lajunen and Summala (2003) assume a tendency towards desirable responding concerning self-reports on driving performance. Participants in our study were not told that an objective point of adaptation was calculated wherefore their impression was that their subjective estimation was the only measurement for their feeling of comfort. Therefore, they could have over-estimated their point of adaptation in order to match with what they thought was expected from them. Moreover, and in line with the phenomenon of a self-enhancement bias (Delhomme, 1991), the over-estimation of one's own driving skills could derive from on an over-confidence in the same, compared to the driving skills of other drivers. As Delhomme (1991) reports, 60 to 90\% drivers claim to drive safer and more skilled than others. Moreover, drivers rate themselves more considerable, reliable and wise than other drivers (McCormick, Walkey, \& Green, 1986), which McKenna, Stanier, and Lewis (1991) try to explain through a "positive self" bias. This bias imputes that drivers possess self-overestimating cognitions perverting their driving skills and causing over-confident beliefs in their performance (Walton \& Bathurst, 1998). Our results add on these findings in that these differences also occur with simulator adaptation and independently of drivers' age, experience in on-road driving and previous adaptation in a training session. As appraising one's driving performance and estimating one's simulator adaptation might be regulated by the same underlying mechanisms of self-appraisal in a driving scenario, our findings are in accordance with Sundström (2008) who reported in her meta-analysis, that driving performance as assessed by self-reports has been shown to be frequently over-estimated in that drivers have overly confident beliefs in their driving skills. Furthermore, Amado, Arıkan, Kaça, Koyuncu, and Turkan (2014) report that $94.8 \%$ of the drivers evaluated their driving skills to be better than an evaluation of an independent observer. Additionally, Amado et al. (2014) show that an over-positive appraisal is higher for drivers who were rated as driving unsafe by independent experts. They suggest that insight into driving performance might be regulated by error and violation indicators in the traffic environment. Consequently, when drivers are not able to process respective feedback from the environment, their ability to assess their driving skills might decrease. A possible explanation for this phenomenon could result from the fact that unsafe drivers take more risks when driving. If they are additionally not involved in road accidents (which is the most likely scenario in driving simulators), this leads to an over-appraisal of driving skills. The same might apply for simulator adaptation, which requires a similar form of self-appraisal during driving. The absence of distracting traffic properties might lead both younger and older drivers to the assumption, that they adapted earlier to the simulator than they actually did. Amado et al. (2014) therefore suppose that feedback might improve self-awareness. In line with this assumption, Roberts, Horrey, and Liang (2016) found that feedback improved awareness of driving performance and in turn, driver calibration within the driving simulator. Although this reveals the possibility of feedback to the drivers, it does yet not ensure

Table 2

Model parameters of the regression analysis.

\begin{tabular}{lllll}
\hline & $\beta$ & $T$ & $P$ \\
\hline Age & 0.131 & 1.87 & 0.062 \\
Driver license possession & 0.464 & 1.29 & 0.198 & 0.4 \\
\hline
\end{tabular}


that subjective estimations of adaptation are valid. Consequently, assessing adaptation to a driving simulator by only asking participants after a training session is not enough to ensure that they actually adapted.

However, our results contradict with findings from Ronen and Yair (2013), supposing that the sensation of having adapted indicated a good measurement for actual adaptation. Differences in findings could be explained by different origins of empirical data. Whereas the calculation of objective adaptation is specifically basing on driving parameters like steering wheel reversals and the lane position, participants were not asked to monitor these parameters when estimating their subjective feeling of having adapted to the simulator. Instead, their estimations of adaptation depend on very individual evaluations of comfort. Hence, both measures depict different approaches to system adaptation which could result in significant differences when comparing them. Moreover, different findings could have emerged due to methodological disparities. It is noteworthy that we artificially completed values in case participants did not objectively adapt to the driving simulator or they did not press the button. In the latter case, there might be other reasons for not having pressed the button than just no subjective adaptation. For instance, older drivers might also be impaired in prospective memory (Salthouse, Berish, \& Siedlecki, 2004) wherefore they could have just forgotten to press the button although they had adapted earlier. Moreover, the usage of different simulated environments is also potentially relevant. For example, McGehee et al. (2004) reported a mean adaptation time of $240 \mathrm{~s}$ for rural roads, whereas Ronen and Yair (2013) identified a time of approximately $540 \mathrm{~s}$ for urban roads (both studies used parameters of steering for quantifying the time of adaptation). While one might assume that the shortest objective adaptation times occur for highways, the present study indicates similar findings as reported by McGehee et al. (2004) for rural roads. We argue that the lack of standardization of simulator scenarios decrease a possible generalization of objective adaptation times, given their relatively low cross-reliability based on methodological differences across studies.

Although we could not identify effects of age and drivers' experience on the discrepancy of subjectively estimated and objectively measured points of adaptation, it is assumable that the discrepancies origin in different backgrounds. While problems of self-appraisal in novice drivers might be due to a lack of driving experience, the discrepancy in the elderly might result from the inadequate portability of real driving experience, cognitive declines, or problems in dealing with virtual environments (for review see Chen \& Chan, 2011). Contrasting evidence comes from Lee (2003) and Lee, Cameron, and Lee (2003) who reported no differences between real on-road driving and simulator driving in older experienced drivers. Based on the findings at hand, drivers of every age and every degree of driving experience seem to be equally inaccurate when estimating the point of time that they subjectively adapt to the driving simulator. A limiting factor of this finding is a distribution of age within the sample that contains mainly very young or very old participants. Middle-aged participants are very rare in this sample. Therefore, further research might try to look for quadratic relationships, as it may be possible that younger drivers show discrepancy in self-assessment due to missing driving experience, whereas older drivers might deviate in self-appraisal due to cognitive declines that come along with aging (Salthouse, 2009). Middle-aged drivers in turn are likely to be experienced drivers, wherefore also a discrepancy in objective and subjective simulator adaptation might decrease.

On the basis of our findings, it seems necessary to make use of objective assessments of simulator adaptation, as this provides a comparable factor between multiple studies and might be an effective tool to provide valid measures. Sundström (2008) states that several studies use multiple different measurements to assess the drivers' subjective driving skills and that methodological problems appear when trying to match subjective and objective driving skills in a simulator. We expand this conclusion by arguing that not only subjective estimates of adaptation but particularly objective measures of adaptation based upon a comprehensive set of performance variables should be assessed to validly define the time needed for a successful adaptation to a driving simulator.

\section{Acknowledgements}

The studies presented here were carried out within the framework of the research project 'ALFASY - Altersgerechte Fahrerassistenzsysteme' ('Age-based Driver Assistance Systems'). This project was funded by the European Regional Development Fund (ERDF).

\section{Appendix A. Supplementary material}

Supplementary data to this article can be found online at https://doi.org/10.1016/j.trf.2019.05.019. 


\section{References}

Amado, S., Arıkan, E., Kaça, G., Koyuncu, M., \& Turkan, B. N. (2014). How accurately do drivers evaluate their own driving behavior? An on-road observational study. Accident Analysis E' Prevention, 63, 65-73. https://doi.org/10.1016/j.aap.2013.10.022.

Bayarri, S., Fernandez, M., \& Perez, M. (1996). Virtual reality for driving simulation. Communications of the ACM, 39(5), 72-77. https://doi.org/10.1145/ 229459.229468.

Boyle, L. N., \& Mannering, F. (2004). Impact of traveler advisory systems on driving speed: Some new evidence. Transportation Research Part C: Emerging Technologies, 12(1), 57-72. https://doi.org/10.1016/j.trc.2003.09.001.

Broen, N. L., \& Chiang, D. P. (1996). Braking response times for 100 drivers in the avoidance of an unexpected obstacle as measured in a driving simulator. Proceedings of the Human Factors and Ergonomics Society Annual Meeting, 40(18), 900-904. https://doi.org/10.1177/154193129604001807.

Chen, K., \& Chan, A. H. (2011). A review of technology acceptance by older adults. Gerontechnology, 10(1), 1-12. https://doi.org/10.4017/ gt.2011.10.01.006.00.

Delhomme, P. (1991). Comparing one's driving with others': Assessment of abilities and frequency of offences. Evidence for a superior conformity of selfbias? Accident Analysis E' Prevention, 23(6), 493-508. https://doi.org/10.1016/0001-4575(91)90015-W.

Desmond, P. A., \& Matthews, G. (1997). Implications of task-induced fatigue effects for in-vehicle countermeasures to driver fatigue. Accident Analysis $\mathcal{E}$ Prevention, 29(4), 515-523. https://doi.org/10.1016/s0001-4575(97)00031-6.

Domeyer, J. E., Cassavaugh, N. D., \& Backs, R. W. (2013). The use of adaptation to reduce simulator sickness in driving assessment and research. Accident Analysis \& Prevention, 53, 127-132. https://doi.org/10.1016/j.aap.2012.12.039.

Dorn, L., \& Barker, D. (2005). The effects of driver training on simulated driving performance. Accident Analysis E' Prevention, 37(1), 63-69. https://doi.org/ 10.1016/j.aap.2004.06.005.

Glendon, A. I., Dorn, L., Davies, D. R., Matthews, G., \& Taylor, R. G. (1996). Age and gender differences in perceived accident likelihood and driver competences. Risk Analysis, 16(6), 755-762. https://doi.org/10.1111/j.1539-6924.1996.tb00826.x.

Godley, S. T., Triggs, T. J., \& Fildes, B. N. (2002). Driving simulator validation for speed research. Accident Analysis E Prevention, 34(5), 589-600. https://doi. org/10.1016/s0001-4575(01)00056-2.

Horberry, T., Anderson, J., \& Regan, M. A. (2006). The possible safety benefits of enhanced road markings: A driving simulator evaluation. Transportation Research Part F: Traffic Psychology and Behaviour, 9(1), 77-87. https://doi.org/10.1016/j.trf.2005.09.002.

Kalbe, E., Kessler, J., Calabrese, P., Smith, R., Passmore, A. P., Brand, M. A., \& Bullock, R. (2004). DemTect: A new, sensitive cognitive screening test to support the diagnosis of mild cognitive impairment and early dementia. International Journal of Geriatric Psychiatry, 19(2), 136-143. https://doi.org/10.1002/ gps.1042.

Kemeny, A., \& Panerai, F. (2003). Evaluating perception in driving simulation experiments. Trends in Cognitive Sciences, 7(1), 31-37. https://doi.org/10.1016/ s1364-6613(02)00011-6.

Kessler, J., Calabrese, P., Kalbe, E., \& Berger, F. (2000). DemTect: A new screening method to support diagnosis of dementia. Psycho, 26(6), 343-347. https:// doi.org/10.1016/s0197-4580(00)82813-4.

Klee, H., Bauer, C., Radwan, E., \& Al-Deek, H. (1999). Preliminary validation of driving simulator based on forward speed. Transportation Research Record, 1689 (1), 33-39. https://doi.org/10.3141/1689-05.

Lajunen, T., \& Summala, H. (2003). Can we trust self-reports of driving? Effects of impression management on driver behaviour questionnaire responses. Transportation Research Part F: Traffic Psychology and Behaviour, 6(2), 97-107. https://doi.org/10.1016/s1369-8478(03)00008-1.

Lee, H. C. (2003). The validity of driving simulator to measure on-road driving performance of older drivers. Transport Engineering in Australia, 8(2), 89-100.

Lee, H. C., Cameron, D., \& Lee, A. H. (2003). Assessing the driving performance of older adult drivers: On-road versus simulated driving. Accident Analysis E Prevention, 35(5), 797-803. https://doi.org/10.1016/s0001-4575(02)00083-0.

Lewis-Evans, B., \& Charlton, S. G. (2006). Explicit and implicit processes in behavioural adaptation to road width. Accident Analysis E Prevention, 38(3), 610-617. https://doi.org/10.1016/j.aap.2005.12.005.

Maas, N., Hesse, B., Koppers, M., \& Schramm, D. (2014). Simulator setup according to use case scenarios-A human-oriented method for virtual development. In 10th international conference on mechatronic and embedded systems and applications (MESA), pp. 1-6. https://doi.org/10.1109/mesa.2014. 6935585.

Maas, N. (2017). Konzeptionierung, Auslegung und Umsetzung von Assistenzfunktionen für die Übergabe der Fahraufgabe aus hochautomatisiertem Fahrbetrieb. Dissertation, University Duisburg-Essen.

Maltz, M., \& Shinar, D. (2004). Imperfect in-vehicle collision avoidance warning systems can aid drivers. Human Factors, 46(2), 357-366. https://doi.org/ 10.1518/hfes.46.2.357.37348.

Matthews, M. L., \& Moran, A. R. (1986). Age differences in male drivers' perception of accident risk: The role of perceived driving ability. Accident Analysis E Prevention, 18(4), 299-313. https://doi.org/10.1016/0001-4575(86)90044-8.

McCormick, I. A., Walkey, F. H., \& Green, D. E. (1986). Comparative perceptions of driver ability-a confirmation and expansion. Accident Analysis E Prevention, 18(3), 205-208. https://doi.org/10.1016/0001-4575(86)90004-7.

McGehee, D. V., Lee, J. D., Rizzo, M., Dawson, J., \& Bateman, K. (2004). Quantitative analysis of steering adaptation on a high performance fixed-base driving simulator. Transportation Research Part F: Traffic Psychology and Behaviour, 7(3), 181-196. https://doi.org/10.1016/s1369-8478(04)00033-6.

McKenna, F. P., Stanier, R. A., \& Lewis, C. (1991). Factors underlying illusory self-assessment of driving skill in males and females. Accident Analysis E Prevention, 23(1), 45-52. https://doi.org/10.1016/0001-4575(91)90034-3.

Owsley, C., \& McGwin, G. Jr, (2010). Vision and driving. Vision Research, 50(23), 2348-2361. https://doi.org/10.1016/j.visres.2010.05.021.

Roberts, S. C., Horrey, W. J., \& Liang, Y. (2016). Measurement of driver calibration and the impact of feedback on drivers' estimates of performance. Accident Analysis \& Prevention, 88, 150-158. https://doi.org/10.1016/j.aap.2015.12.013.

Ronen, A., \& Yair, N. (2013). The adaptation period to a driving simulator. Transportation Research Part F: Traffic Psychology and Behaviour, 18, 94-106. https:// doi.org/10.1016/j.trf.2012.12.007.

Salthouse, T. A., Berish, D. E., \& Siedlecki, K. L. (2004). Construct validity and age sensitivity of prospective memory. Memory E Cognition, 32(7), 1133-1148. https://doi.org/10.3758/bf03196887.

Salthouse, T. A. (2009). When does age-related cognitive decline begin? Neurobiology of Aging, 30(4), 507-514. https://doi.org/10.1016/j. neurobiolaging.2008.09.023.

Salvucci, D. D. (2001). Predicting the effects of in-car interface use on driver performance: An integrated model approach. International Journal of HumanComputer Studies, 55(1), 85-107. https://doi.org/10.1006/ijhc.2001.0472.

Schweig, S., Liebherr, M., Schramm, D., Brand, M. \& Maas, N. (2018). The impact of psychological and demographic parameters on simulator sickness. In Proceedings of 8th international conference on simulation and modeling methodologies, technologies and applications, 1, pp. 91-97. https://doi.org/10. $5220 / 0006837300910097$.

Sundström, A. (2008). Self-assessment of driving skill-A review from a measurement perspective. Transportation Research Part F: Traffic Psychology and Behaviour, 11(1), 1-9. https://doi.org/10.1016/j.trf.2007.05.002.

Takayama, L., \& Nass, C. (2008). Driver safety and information from afar: An experimental driving simulator study of wireless vs. in-car information services. International Journal of Human-Computer Studies, 66(3), 173-184. https://doi.org/10.1016/j.ijhcs.2006.06.005.

Underwood, G., Crundall, D., \& Chapman, P. (2011). Driving simulator validation with hazard perception. Transportation Research Part F: Traffic Psychology and Behaviour, 14(6), 435-446. https://doi.org/10.1016/j.trf.2011.04.008.

Walton, D., \& Bathurst, J. (1998). An exploration of the perceptions of the average driver's speed compared to perceived driver safety and driving skill. Accident Analysis E Prevention, 30(6), 821-830. https://doi.org/10.1016/S0001-4575(98)00035-9.

Weinberg, G., \& Harsham, B. (2009). Developing a low-cost driving simulator for the evaluation of in-vehicle technologies. AutomotiveUI, 9, 51-54. https:// doi.org/10.1016/j.trf.2011.04.008. 